\title{
Serotonin-Induced Cleavage of the Atypical Protein Kinase C Apl III in Aplysia
}

\author{
Joanna K. Bougie, ${ }^{1}$ Diancai Cai, ${ }^{3}$ Margaret Hastings, ${ }^{1}$ Carole A. Farah, ${ }^{2}$ Shanping Chen, ${ }^{3}$ Xiaotang Fan, ${ }^{2}$ \\ Patrick K. McCamphill, ${ }^{2}$ David L. Glanzman, ${ }^{3,4,5,6}$ and Wayne S. Sossin ${ }^{1,2}$ \\ ${ }^{1}$ Department of Psychology, McGill University, Montreal Neurological Institute, Montreal, Quebec H3A 1B1, Canada, ${ }^{2}$ Department of Neurology and \\ Neurosurgery, Montreal Neurological Institute, McGill University, Montreal, Quebec H3A 2B4, Canada, ${ }^{3}$ Department of Integrative Biology and Physiology, \\ University of California, Los Angeles, College of Letters and Science, ${ }^{4}$ Department of Neurobiology, ${ }^{5}$ Brain Research Institute, and ${ }^{6}$ Integrative Center for \\ Learning and Memory, David Geffen School of Medicine, University of California, Los Angeles, Los Angeles, California 90095
}

A constitutively active kinase, known as protein kinase $\mathrm{M} \zeta(\mathrm{PKM} \zeta)$, is proposed to act as a long-lasting molecular memory trace. While $\mathrm{PKM} \zeta$ is formed in rodents through translation of a transcript initiating in an intron of the protein kinase $\mathrm{C} \zeta$ (PKC $\zeta$ ) gene, this transcript does not exist in Aplysia californica despite the fact that inhibitors of PKM $\zeta$ erase memory in Aplysia in a fashion similar to rodents. We have previously shown that, in Aplysia, the ortholog of PKC $\zeta$, PKC Apl III, is cleaved by calpain to form a PKM after overexpression of PKC Apl III. We now show that kinase activity is required for this cleavage. We further use a FRET reporter to measure cleavage of PKC Apl III into PKM Apl III in live neurons using a stimulus that induces plasticity. Our results show that a 10 min application of serotonin induces cleavage of PKC Apl III in motor neuron processes in a calpain- and protein synthesis-dependent manner, but does not induce cleavage of PKC Apl III in sensory neuron processes. Furthermore, a dominant-negative PKM Apl III expressed in the motor neuron blocked the late phase of intermediate-term facilitation in sensory-motor neuron cocultures induced by 10 min of serotonin. In summary, we provide evidence that PKC Apl III is cleaved into PKM Apl III during memory formation, that the requirements for cleavage are the same as the requirements for the plasticity, and that PKM in the motor neuron is required for intermediate-term facilitation.

\section{Introduction}

Experience induces changes in the strength of synapses, and this synaptic plasticity is thought to be the cellular basis for learning and memory (Si et al., 2003). Moreover, the length of time a memory lasts is related to the molecular memory traces formed by the experience (Sossin, 2008). In particular, evidence suggests that production of a constitutively active kinase, known as protein kinase $\mathrm{M} \zeta(\mathrm{PKM} \zeta)$, acts as a molecular memory trace. In rodents, $\mathrm{PKM} \zeta$ is necessary and sufficient for the maintenance phase of long-term potentiation (LTP), a cellular model for behavioral memory formation (Serrano et al., 2005; Pastalkova et al., 2006). Furthermore, inhibitors of PKM $\zeta$ are able to reverse in vivo LTP and produce persistent loss of behavioral memory at time points after memories are presumed to be consolidated (Ling et al., 2002; Serrano et al., 2005; Shema et al., 2007, 2011).

Received June 15, 2011; revised Aug. 21, 2012; accepted Aug. 26, 2012.

Author contributions: J.K.B., D.L.G., and W.S.S. designed research; J.K.B., D.C., M.H., C.A.F., S.C., X.F., and W.S.S. performed research; C.A.F. contributed unpublished reagents/analytic tools; J.K.B., D.C., M.H., P.K.M., D.L.G., and W.S.S. analyzed data; J.K.B., C.A.F., D.L.G., and W.S.S. wrote the paper.

This work was supported by Canadian Institutes of Health Research Grant MOP 12046 (W.S.S.). J.K.B. is the recipient of a doctoral fellowship from the Fonds de la Recherche en Santé du Québec (FRSQ), and M.H. and P.K.M. were supported by fellowships from the National Science and Engineering Research Council of Canada. W.S.S. is a James McGill Professor and a FRSQ Chercheur National.

Correspondence should be addressed to Wayne S. Sossin, Department of Neurology and Neurosurgery, Montreal Neurological Institute, McGill University, 3801 University Street, Montreal, Quebec H3A-2B4, Canada. E-mail: wayne.sossin@mcgill.ca.

DOI:10.1523/JNEUROSCI.3026-11.2012

Copyright $\odot 2012$ the authors $\quad 0270-6474 / 12 / 3214630-11 \$ 15.00 / 0$
We use the invertebrate Aplysia californica as a model system for examining molecular memory traces. Aplysia has a number of advantages: the specific synapses that are known to be modified by experience and thus encode memory have been identified, and Aplysia nerve cells are large and ideal for cellular imaging studies. In Aplysia, noxious stimuli induce the release of the neurotransmitter serotonin (5-HT) that in turn leads to the strengthening (facilitation) of the sensory-motor neuron synapses (Marinesco and Carew, 2002). The increased synaptic connection contributes to the behavioral sensitization to the stimulus (Kandel, 2001). Another major advantage of this system is that facilitation can be recapitulated in sensory-motor neuron cultures after addition of 5-HT (Montarolo et al., 1986), allowing for the examination of the formation of molecular traces in a tractable culture situation. Many of the signaling pathways underlying memory formation were first characterized in this system (Roberts and Glanzman, 2003).

Recently, it has been demonstrated that, similar to results in rodents, inhibitors of $\mathrm{PKM} \zeta$ reverse consolidated synaptic facilitation and long-term memory in Aplysia, suggesting a conserved role for an atypical PKM in formation of the memory trace (Cai et al., 2011). However, in rodents, $\mathrm{PKM} \zeta$ is generated by translation of a unique mRNA whose transcription starts in an intron of protein kinase $\mathrm{C} \zeta$ (PKC $\zeta$ ) (Hernandez et al., 2003). This transcript does not exist in species below chordates (Bougie et al., 2009). Instead, we have postulated that calpain-induced cleavage produces a PKM from the PKC $\zeta$ ortholog in Aplysia (PKC Apl III), which we named PKM Apl III (Bougie et al., 2009; Villareal et al., 2009). 
In the present study, we characterize a FRET reporter to measure cleavage of PKC Apl III in living neurons and use it to demonstrate 5-HT-mediated, calpain-dependent, cleavage of PKC Apl III in the motor neuron. We find cleavage of PKC Apl III in response to overexpression requires kinase activity, suggesting a putative positive-feedback model in which initial calpain cleavage produces a PKM that can then induce additional calpain activation. Moreover, a dominant-negative form of PKM Apl III expressed in the motor neuron can block intermediate-term facilitation (ITF) induced by a $10 \mathrm{~min}$ application of 5-HT.

\section{Materials and Methods}

Animals. Aplysia californica (75-125 g) were obtained from Marine Specimens Unlimited and the Mariculture Facility of the University of Miami (Miami, FL). The animals were then maintained in a salt water aquarium until experimentation.

Constructs. The monomeric red fluorescent protein (mRFP)-PKC Apl III and mRFP-PKM Apl III were previously described (Bougie et al., 2009). The kinase-dead mRFP-PKC Apl III D-A and mRFP-PKM Apl III D-A were made by mutating the aspartic acid 392 in mRFP-PKC Apl III and mRFP-PKM Apl III to alanine using overlap PCR (D392A). To make the cyan fluorescent protein (CFP)-PKC Apl III-yellow fluorescent protein (YFP) FRET construct, enhanced CFP (eCFP) was amplified by PCR using primers containing SphI and XhoI sites. The product of this amplification was then cut with SphI and XhoI and used to replace the mRFP from the aforementioned mRFP-PKC Apl III construct cut with these same enzymes. Enhanced YFP (eYFP) was then amplified by PCR using primers containing Nco and Blpl sites with the nucleotides encoding a putative PDZ binding domain (MSMEDCV) at the end of PKC Apl III added on at the $3^{\prime}$ end. The product of this amplification was then cut with Nco and Esp1 and ligated to the CFP-PKC Apl III vector cut with the same enzymes. Baculovirus expression constructs were generated using the Invitrogen Bac-to-Bac cloning system according to the manufacturer's instructions.

Protein purification. SF9 cells in suspension were infected with baculovirus constructs as previously described (Lim et al., 2006). Three days after infection, His-tagged protein was purified using Invitrogen Probond His-Affinity resin (Invitrogen), in modified purification buffer (20 mM HEPES, pH 7.5, $10 \mathrm{~mm} \mathrm{MgCl}_{2}, 1$ mм DTT, 100 mм KCl, 10\% glycerol; for calpains: 20 mм HEPES, pH 7.5, 1 mм EDTA, 1 mм DTT, 100 $\mathrm{mm} \mathrm{KCl,} 10 \%$ glycerol). Proteins were eluted in elution buffer (identical with purification buffer but with $0.25 \mathrm{~m}$ imidazole), DTT was added to a final concentration of $10 \mathrm{~mm}$, and the sample was concentrated using an Amicon Ultra centrifugal filter and stored at $-80^{\circ} \mathrm{C}$.

Antibodies. The C-terminal and phosphospecific antibodies were previously described (Bougie et al., 2009). Either an Alexa 647 goat antirabbit secondary (Invitrogen) at a concentration of 1:200, or a FITC goat anti-rabbit green secondary antibody (Zymed) at a concentration of 1:100, was used to visualize the primary antibodies.

Aplysia cell culture and DNA microinjection. Aplysia dissociated sensory and motor neuron cultures were prepared according to the protocol outlined in the study by Zhao et al. (2006), with slight modifications. The ganglia were digested for either $2 \mathrm{~h}$ at $37^{\circ} \mathrm{C}$, or at $19^{\circ} \mathrm{C}$ for $18-19 \mathrm{~h}$ in 10 $\mathrm{mg} / \mathrm{ml}$ dispase. Individual neurons were pulled from desheathed pleural (for sensory neurons) or abdominal ganglia [for siphon (LFS) motor neurons] and isolated in Leibowitz-15 (L-15) media (Sigma-Aldrich; supplemented with $0.2 \mathrm{M} \mathrm{NaCl}, 26 \mathrm{~mm} \mathrm{MgSO}_{4} \cdot 7 \mathrm{H}_{2} \mathrm{O}, 35 \mathrm{~mm}$ dextrose, 27

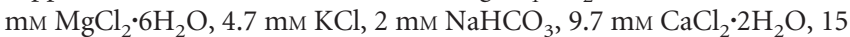
mM HEPES, and the $\mathrm{pH}$ was adjusted to 7.4) containing 25-50\% Aplysia hemolymph. Cells were then plated either on coverslips $(0.16-0.19 \mathrm{~mm})$ or on MatTek glass bottom culture dishes (MatTek Corporation) with a glass surface of $14 \mathrm{~mm}$ and a coverslip thickness of $0.16-0.19 \mathrm{~mm}$, pretreated with poly-L-lysine (BD Biosciences).

Solutions of the construct of interest at various concentrations were prepared in filtered double-distilled water and $0.5 \%$ fast green dye. The solutions were loaded into glass micropipettes, and the tip of the micropipette was then inserted into the cell nucleus. Short pressure pulses were delivered until the nucleus became uniformly green. Following injection, the cells were kept at $19^{\circ} \mathrm{C}$ and treatment with various reagents was performed $1-3 \mathrm{~d}$ after injection. The cells were then fixed and stained for immunocytochemistry or were imaged live for the FRET experiments.

Immunocytochemistry. Immunocytochemistry was performed as previously described (Bougie et al., 2009).

SF9 cell culture. The SF9 cells were purchased from Sigma-Aldrich. SF9 cells were grown in Grace's medium (Invitrogen) supplemented with $10 \%$ fetal bovine serum (Cansera) as a monolayer at $27^{\circ} \mathrm{C}$. For transfection, cells were plated on MatTek glass bottom culture dishes (MatTek Corporation) with a glass surface of $14 \mathrm{~mm}$ and a coverslip thickness of $0.16-0.19 \mathrm{~mm}$. Cells were transfected using the Cellfectin reagent (Invitrogen) following the recommendation of the manufacturer (Zhao et al., 2006).

Determination of specific activity. The CFP-PKC Apl III-YFP, WT PKC Apl III, and mRFP-PKC D392A kinases were purified from SF9 cells infected with baculovirus encoding the different PKC isoforms as described previously (Lim and Sossin, 2006). Kinase assays were performed as described previously (Lim and Sossin, 2006), and a serial dilution of enzyme ensured that the assay was in the linear range. All the constructs contained an HA tag and the relative amount of enzyme was quantified by immunoblotting a serial dilution of enzymes from the same aliquot of purified enzyme used in the enzyme assay with the HA antibody. All values were normalized to the WT PKC Apl III measured in that experiment. Specific activity was determined by dividing the activity by the amount of enzyme.

In vitro calpain assay. Aplysia PKC Apl III with or without CFP and YFP tags were purified from SF9 cells infected with baculovirus encoding the different isoforms as described previously (Lim and Sossin, 2006). Calpain-1 purified from porcine erythrocytes (Calbiochem) was incubated with the recombinant Aplysia PKC Apl III with or without CFP and YFP tags for $30 \mathrm{~min}$ at $30^{\circ} \mathrm{C}$ in calpain activation buffer (final concentrations: $5 \mathrm{~mm}$ L-cysteine, $100 \mathrm{~mm}$ imidazole, $5 \mathrm{~mm}$ calcium). Reaction products were then subjected to SDS-PAGE followed by immunoblotting. The membrane was probed with a mouse monoclonal antibody to the His-tag (Invitrogen). Cleavage was quantified as the loss of the fulllength protein.

FRET and image quantification. Twenty-four hours following DNA microinjection, the cells were imaged using a Zeiss fluorescent microscope. Images of cells expressing eCFP alone and eYFP alone were taken as control measures for bleed-through subtraction. Each cell was imaged in the CFP, YFP, and FRET configurations in which exposure times were kept constant for all groups within each experiment. For the sensory and motor neuron experiments in which CFP-PKC Apl III-YFP was expressed at low levels, the FRET channel exposure times were doubled to yield visible FRET levels for quantification. This improves the signal-tonoise ratio and does not affect our ability to detect changes in the FRET signal over time. Zeiss AxioVision software was used to quantify the images, where CFP is assigned channel 1, YFP is assigned channel 2, and FRET is assigned channel 3. The FRET Xia formula was used to subtract spectral bleed-through from cyan and yellow channels (Xia and Liu, 2001). The end result is a FRET ratio ranging from 0 to 1 , expressed as a corresponding color-coded FRET ratio map in which lower levels of FRET are assigned cooler colors and higher levels of FRET are assigned warmer colors.

To determine changes induced by 5-HT, the FRET maps were coded so that the person doing the quantification was unaware of the treatment, or the time (pre vs post) for the image. NIH Image was then used to outline a large region of interest in the process, and this area was measured in both the pre and post image. The fold change in signal was then calculated (post/pre). In most cases, multiple pictures of each cell (two to three) and multiple regions of interest (two to three) were chosen, and the average of the fold changes was used to measure the 5-HT-induced change in FRET for each treated cell.

Confocal microscopy and image quantification. Neurons expressing the mRFP constructs were imaged using the 510 or 710 Zeiss laser-scanning microscope, and pictures were captured using the $40 \times$ objective at the middle section of the cell where the nucleus was as well defined as possible. Within each experiment, the same laser power was used for all 
A
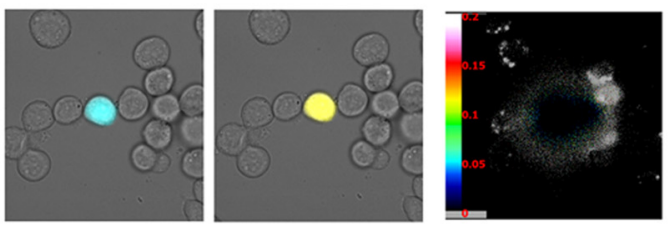

$C F P+Y F P$
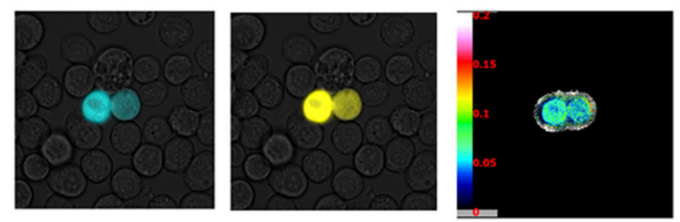

CFP-PKC ApI III-YFP

$\mathrm{B} \equiv$

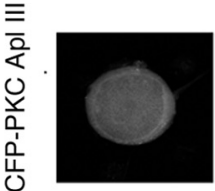

CFP

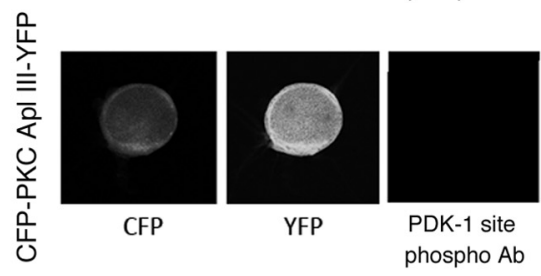

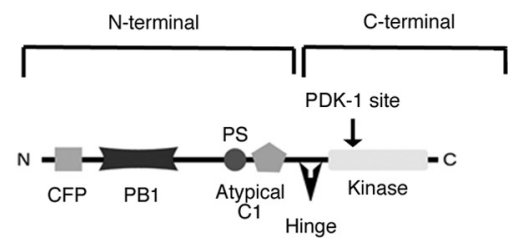

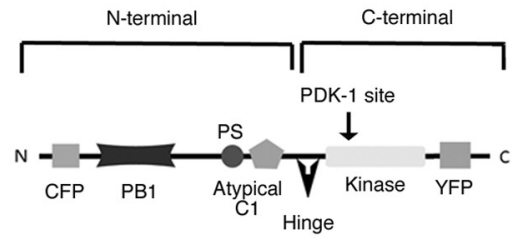

$\mathrm{D}$

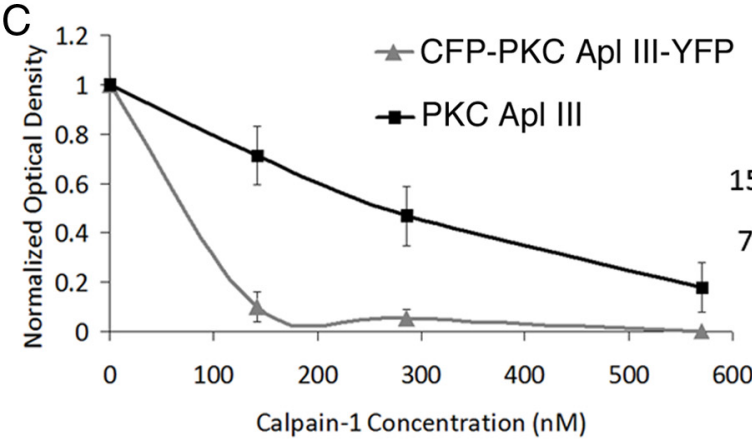

CFP-PKC Apl III-YFP PKC Apl III

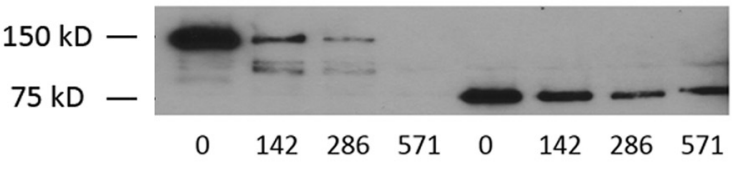

Calpain-1 Concentration (nM)

Figure 1. Characterization of a FRET reporter, CFP-PKC ApI III-YFP. A, Transfected SF9 cells: Top row, Cotransfection of CFP and YFP in same cell; bottom row, CFP-PKC Apl III-YFP. Images were captured using a Zeiss fluorescent microscope. The FRET maps display color-coded images of the measured FRET signal, in which warm colors represent higher levels of FRET and cooler colors represent lower levels of FRET (the gray color is unassigned and represents negative or undefined values sometimes seen in the periphery of expressing cells, or when no FRET is observed). The FRET maps display detectable FRET for CFP-PKC Apl III-YFP (FRET, 0.0651 for cell on left; FRET, 0.0638 cell on right) while no FRET for CFP and YFP in the same cell (FRET, 0.0000 ). FRET map scale ranges from 0.00 to 0.20. B. Aplysia sensory neurons expressing CFP-PKC ApI III (top row) or CFP-PKC ApI III-YFP (bottom row) were stained with the phosphospecific antibody (Bougie et al., 2009), and images were captured with a Zeiss confocal microscope; laser power was kept the same between all conditions. Representative diagrams of the constructs are shown on the right, where the CFP is located on the N terminus, and YFP and/or antibody epitope (PDK-1 site) is on the C terminus. The domains of the atypical PKC are shown (PS, pseudosubstrate). C, Quantification of calpain assay performed from purified proteins from baculovirus-infected SF9 cells expressing CFP-PKC ApI III-YFP or PKC ApI III with no fluorescent tags. The percentage of the full-length protein remaining was measured by optical density readings ( $y$-axis) and plotted against increasing concentrations of mammalian calpain-1 ( $x$-axis). Error bars indicate SEM. D, Representative blot of the calpain assay, purified CFP-PKC ApI III-YFP and PKC ApI III were probed with anti-His-antibody targeting His-tag at N terminus of each protein with increasing amounts of mammalian calpain-1 (0, 142, 286, and $571 \mathrm{~nm}$, respectively). The full-length protein (largest band) disappears with increased levels of calpain. CFP-PKC ApI III-YFP runs at a molecular weight of $150 \mathrm{kDa}$, whereas the untagged PKC has a molecular weight of $75 \mathrm{kDa}$.

groups, unless otherwise described. Quantification was performed as previously described (Bougie et al., 2009). Briefly, all cells including control cells were normalized to the average ratio of the control cells in that experiment. These normalized values were then combined from the different experiments.

Electrophysiology. Sensory-motor cocultures, each comprising a single presynaptic sensory neuron and a single postsynaptic motor neuron, were fabricated using LFS motor neurons and pleural sensory neurons. The culture medium contained 50\% Aplysia hemolymph and 50\% L-15 (supplemented as described above). The cultures were maintained at $18^{\circ} \mathrm{C}$ for $2 \mathrm{~d}$ before the microinjection of DNA constructs. Solutions of plasmid constructs of either dominant-negative mRFP-PKC Apl III (DN-Apl III) or mRFP alone in $\mathrm{dH}_{2} \mathrm{O}$ with $0.25 \%$ fast green were pressure-injected into the cell nucleus of postsynaptic motor neurons. After overnight incubation at $18^{\circ} \mathrm{C}$, the cocultures were imaged using a Zeiss laser-scanning confocal microscope to confirm the expression of mRFP constructs. During experiments, the cultures were perfused with $50 \%$ sterile artificial seawater (ASW) and 50\% L-15. All experiments were performed at room temperature $\left(18-22^{\circ} \mathrm{C}\right)$. The electrophysiological stimulating and recording methods were as described previously ( $\mathrm{Li}$ et al., 2005). Synaptic strength was determined by eliciting a single EPSP in the motor neuron via intracellular activation of the sensory neuron. 
Immediately after impalement of the sensory neuron and motor neuron, the sensory neuron was fired once and the size of the EPSP was determined. After this initial test, there was a period of $30 \mathrm{~min}$ before the start of the experiment. If the size of the EPSP declined by 50\% during this period, the experiment was discontinued. The EPSPs were recorded once per 5 min during the experiments. 5-HT was prepared fresh daily as a 2 mM stock solution dissolved in ASW. The 5-HT was diluted to a final concentration of $20 \mu \mathrm{M}$ in perfusion medium just before an experiment and applied to the cultured synapses for $10 \mathrm{~min}$, after which it was rapidly washed out with normal perfusion medium.

The peak amplitude of the EPSP was normalized to the amplitude of the EPSP at 0 min for the same coculture. The normalized data were expressed as means \pm SEM. Statistical comparisons were performed using SPSS (IBM). For multiple-group comparisons, repeated-measures ANOVAs were first performed, followed by between-group comparisons with Tukey's HSD post hoc tests. The two experimental trials during which 5-HT was present in the bath (the 10 and 15 min trials) were treated separately from the trials that occurred after washout of the drug. Therefore, two repeated-measures ANOVAs were performed: one for the two trials performed in 5-HT and the other for the trials (20-55 min trials) following 5-HT washout. All reported levels of statistical significance represent two-tailed values.

\section{Results}

Characterization of a FRET reporter, CFP-PKC Apl III-YFP

To monitor persistent activation by cleavage of PKC Apl III during plasticity, we generated a construct with a CFP located at the $\mathrm{N}$ terminus and a YFP located at the $\mathrm{C}$ terminus (CFP-PKC Apl III-YFP). This construct is expected to show a FRET signal due to proximity and structural configuration of the CFP and YFP in the kinase (Xia and Liu, 2001). Cleavage of CFP-PKC Apl III-YFP could then be measured by a loss of this FRET signal. We first transfected SF9 cells with either the CFP-PKC Apl III-YFP FRET construct or cotransfected CFP and YFP together as a negative control. The cells were then imaged for FRET (see Materials and Methods). As expected, the CFP-PKC Apl III-YFP construct has a measurable FRET signal, while no FRET signal could be detected in cells expressing both CFP and YFP (Fig. 1A).

We have previously shown that overexpression of mRFP-PKC Apl III in sensory neurons or motor neurons led to cleavage and differential localization of the $\mathrm{N}$ terminus (nucleus and cytoplasm) and C terminus (cytoplasm) of the protein (Bougie et al., 2009). This difference reflects a nuclear localization site in the $\mathrm{N}$ terminus that leads to nuclear-cytoplasmic cycling of the $\mathrm{N}$ terminus, and restriction of the $\mathrm{C}$ terminus to the cytoplasm following cleavage due to the loss of the nuclear localization site (Bougie et al., 2009). However, the CFP-PKC Apl III-YFP construct did not show this pattern of dissociation in sensory neurons, as both the fluorescence from CFP and the fluorescence from YFP showed nuclear as well as cytoplasmic staining at all levels of expression (Fig. $1 B$ ) (data not shown). Similar results were seen in motor neurons (data not shown). Since overexpression-dependent cleavage is observed both in isolated sensory and motor neurons, we have examined this process in sensory neurons, since there are many more sensory neurons per animal than motor neurons.

One possible explanation for the lack of separation of the $\mathrm{N}$ and $\mathrm{C}$ termini was that the addition of the fluorescent reporter proteins blocked the cleavage of PKC Apl III by calpain. To test this, we generated a baculovirus expressing the CFP-PKC Apl III-YFP with a His-tag at the $\mathrm{N}$ terminus and purified the protein from baculovirus-infected SF9 cells. We then compared the cleavage of this construct and PKC Apl III by mammalian calpains and found that the CFP-PKC Apl III-YFP was actually cleaved somewhat more efficiently than PKC Apl III (Fig. 1C), ruling out the possibility that the different distribution seen in
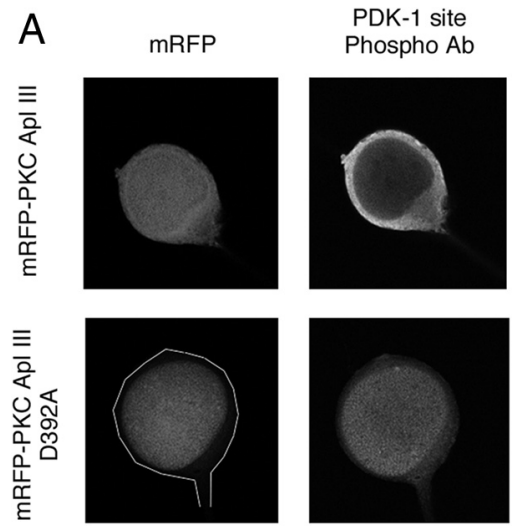

B
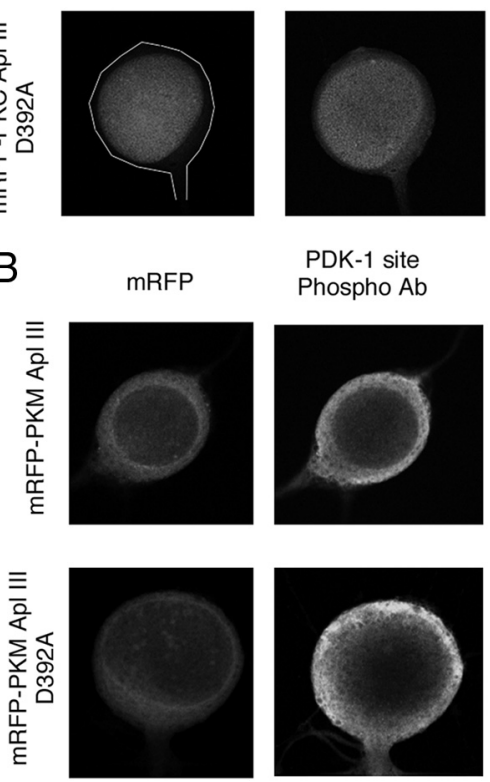

Figure 2. Kinase activity is required for cleavage and nuclear export of PKC ApI III. A, Aplysia sensory neurons expressing mRFP-PKC ApI III (top) or mRFP-PKC ApI III D392-A (bottom) were imaged for the $\mathrm{N}$ terminus (mRFP fluorescence; $\mathrm{mRFP}$ ) and ( terminus [immunoreactivity with the anti-PDK1 phosphospecific antibody (PDK-1 site Phospho Ab)]. All cells were visualized using a Zeiss laser-scanning confocal microscope with laser power kept constant between conditions. Outlines are drawn around cells when the cytoplasmic staining is difficult to see. When mRFP_PKC ApI III D392A is expressed, both mRFP fluorescence and immunoreactivity for the anti-PDK1 phosphospecific antibody show increased nuclear localization compared with when mRFP_PKC Apl III is expressed. Results are given in the text. $B$, Aplysia sensory neurons expressing mRFP-PKM Apl III (top) and mRFP-PKM D392A Apl III (bottom) were imaged for the N terminus (mRFP fluorescence; $m R F P$ ) and C terminus [immunoreactivity with the anti-PDK1 phosphospecific antibody (PDK-1 site Phospho Ab)]. All cells were visualized using a Zeiss laserscanning confocal microscope with laser power kept constant between conditions. Both constructs showed mainly cytoplasmic localization for both $\mathrm{N}$-terminal and C-terminal markers.

neurons was due to a blockade of cleavage sites in the tagged kinase. We then assayed the purified kinases for kinase activity and found that the CFP-PKC Apl III-YFP had less specific activity than PKC Apl III ( $10 \pm 1 \%$ of the PKC Apl III activity; $n=3)$. This suggests that the addition of the YFP at the $\mathrm{C}$ terminus of the protein lowered the specific activity of the kinase. It is known that PKC Apl III is activated by phosphorylation at the PDK1 site (Chou et al., 1998). To directly compare the impact of adding the YFP to the C-terminal side on PDK1 phosphorylation, we compared phosphorylation of CFP-PKC Apl III to the CFP-PKC Apl III-YFP after expression in sensory neurons. Consistent with a decrease in activity after addition of the YFP tag, there was much less staining of the CFP-PKC Apl III-YFP with the phosphospecific antibody to the PDK-1 site, despite the comparable expression levels (visualized by CFP staining) (Fig. $1 B$ ). If the lack of cleavage of CFP-PKC Apl III-YFP was due to its decreased activity, it suggests that overexpression-dependent cleavage of PKC Apl III might be due to kinase activation of a proteolytic event. 
Kinase activity is required for overexpression-induced cleavage of PKC Apl III

To test the hypothesis that kinase activity is required for cleavage of PKC Apl III after overexpression, we generated a kinasedead PKC in a distinct manner. A previously established problem that arises with kinase-dead PKCs is that the kinases are not folded correctly due to a lack of phosphorylation at key sites. It has been shown that mutation of a conserved aspartate (D532 in $\mathrm{PKC} \varepsilon$ ) to alanine leads to a kinase-dead PKC that still maintain integrity of the ATP binding pocket but make no side interactions with ATP. Thus, this mutation leads to PKCs that are kinase-dead but retain stability of the active conformer, and are still able to be phosphorylated and constitutively primed (Cameron et al., 2009). This residue is conserved in PKC Apl III (D392). Thus, we made this mutation in the mRFP-PKC Apl III (mRFP-PKC Apl III D392A) and expressed this construct in sensory neurons. Unlike the CFP-PKC Apl III-YFP (Fig. 1B), and a previous kinase-dead mutant we had generated (mRFP-PKC Apl III K297R) (data not shown), the mRFP-PKC Apl III D392A was phosphorylated at the PDK1 site (Fig. 2A). Despite this, the kinase activity of the purified mRFP-PKC Apl III D392A was extremely low $(3 \pm 3 \% ; n=3$ of the PKC Apl III activity).

Consistent with a requirement of kinase activity for cleavage, mRFP-PKC Apl III D392A showed little evidence for the differential localization of the $\mathrm{N}$ terminus (measured by mRFP fluorescence) and C terminus (measured using immunostaining of the phosphospecific antibody to the PDK-1 site in the kinase domain) that is seen with mRFP-PKC Apl III (Fig. 2A). This is quantified by the increased nuclear/ cytoplasmic ratio of the immunostaining for the C-terminal marker (phosphospecific antibody to the PDK-1 site) for mRFP-PKC Apl III D392A compared with mRFP-PKC Apl III (1.22 \pm 0.08 for mRFP-PKC Apl III D392A; $n=27$ sensory neurons from three independent experiments compared with $0.52 \pm 0.04$ for mRFP-PKC Apl III; $n=31$ sensory neurons from three independent experiments) ( $p<0.001$, Student's $t$ test). We were surprised to find that there was also an increased nuclear/cytoplasmic ratio observed for mRFP fluorescence for mRFP-PKC Apl III D392A compared with mRFP-PKC Apl III (Fig. $2 A ; 2.70 \pm 0.21$ for mRFP-PKC Apl III D392A compared with $0.97 \pm 0.02$ for mRFP-PKC Apl III; $p<0.001$, Student's $t$ test). This suggests that kinase activity is also required for nuclear export of PKC Apl III. We confirmed the requirement for PKM (results in text).
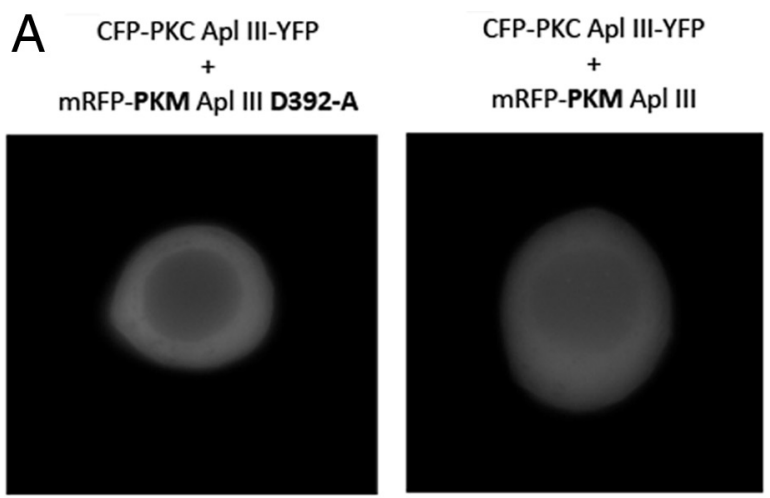

\section{mRFP}
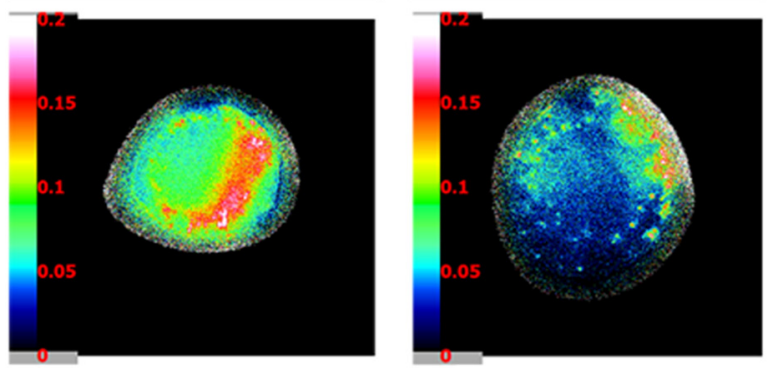

FRET

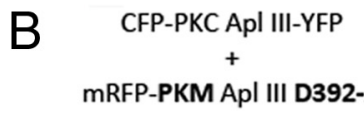

CFP-PKC Apl III-YFP $+$ mRFP-PKM Apl III
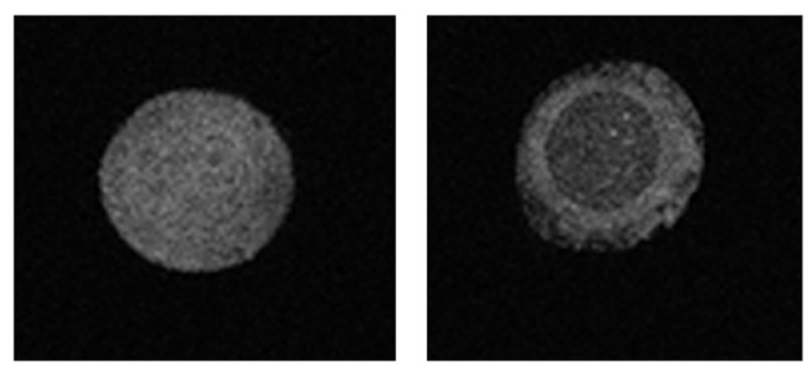

\section{CFP}
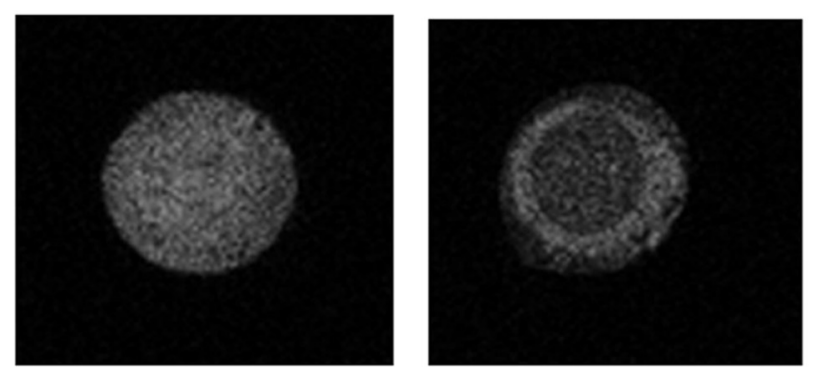

YFP

Figure 3. Increasing kinase activity is sufficient to induce cleavage and nuclear export of PKC Ap III. A, Aplysia sensory neurons were coinjected with CFP-PKC ApI III-YFP and either mRFP-PKM ApI III D392A (left) or mRFP-PKM Apl III (right). mRFP fluorescence was measured to gauge expression levels (top), and FRET Xia levels were measured and shown using a color-coded FRET map of the measured FRET ratio (scale, 0.0 to 0.2 ) (bottom). The FRET maps display color-coded images of the measured FRET signal, where warm colors represent higher levels of FRET and cooler colors represent lower levels of FRET (the gray color is unassigned and represents negative or undefined values sometimes seen in the periphery of expressing cells, or when no FRET is observed). FRET values are lower when active PKM is expressed (results in text). $\boldsymbol{B}$, Aplysia sensory neurons coinjected with CFP-PKC ApI III-YFP and either mRFP-PKM Apl III D392A Apl III (left) or mRFP-PKM (right). Images are of the N-terminal marker, CFP fluorescence (CFP) (top) or C-terminal marker, YFP fluorescence (YFP) (bottom). Both CFP and YFP are more cytoplasmic after expression of the active

kinase activity for the redistribution of the C terminus of PKC Apl III using the PKC inhibitor chelerythrine $(10 \mu \mathrm{M})$. When we added the inhibitor immediately following injection of the plasmid encoding mRFP-PKC Apl III, we were able to replicate the lack of overexpression dependent redistribution of the $\mathrm{C}$ terminus to the cytoplasm 

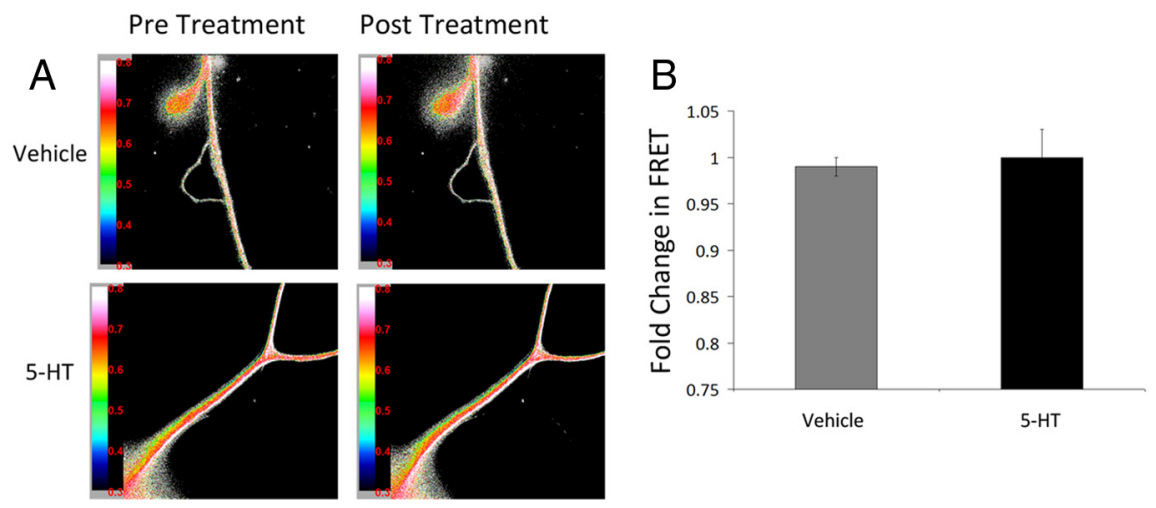

Figure 4. 5-HT does not induce cleavage of CFP-PKC ApI III-YFP in Aplysia sensory neuron processes. $A$, Color-coded FRET maps of Aplysia motor neuron processes expressing low levels of CFP-PKC ApI III-YFP, pretreatment and posttreatment. Scale is from 0.3 to 0.8. The FRET maps display color-coded images of the measured FRET signal, in which warm colors represent higher levels of FRET and cooler colors represent lower levels of FRET (the gray color is unassigned and represents negative or undefined values sometimes seen in the periphery of expressing cells, or when no FRET is observed). No change with 5 - $\mathrm{HT}$ is observed. $\boldsymbol{B}$, Quantification of $\boldsymbol{A}$ : Fold change in FRET was calculated for cells treated with a vehicle solution $(n=13$ cells from three independent experiments) or $5-\mathrm{HT}$ ( $n=18$ cells from three independent experiments). $p>0.05$, Student's $t$ test. Error bars indicate SEM.

(nuclear/cytoplasmic ratio for phosphospecific antibody to the PDK-1 site after chelerythrine treatment was $1.20 \pm 0.06 ; n=41$ cells from three independent experiments similar to the ratio seen above with the kinase-dead mRFP-PKC Apl III D392A, $1.22 \pm 0.08$ and different from the ratio seen with the kinase active mRFP-PKC Apl III in the absence of the PKC inhibitor, $0.52 \pm 0.04$ ). Chelerythrine treatment also greatly decreased the mRFP fluorescence, probably due to quenching, making quantifying the mRFP fluorescence difficult (data not shown). This was not specific to the mRFP-PKC Apl III, as fluorescence of mRFP alone was also greatly quenched after treatment with chelerythrine (data not shown).

The increased nuclear localization of mRFP-PKC Apl III D392A implies that kinase activity is required for efficient nuclear export. It is also possible that conformational changes induced by the mutation that altered accessibility to nuclear import or export signals independently of the loss of kinase activity could explain this distribution. To distinguish between these possibilities, we generated an mRFP-PKM Apl III D392A, a construct lacking the regulatory domain and thus also lacking the putative nuclear import site. It was therefore predicted that this construct would be localized to the cytoplasm. However, if the D-A mutation caused a conformational change that caused nuclear retention through some other mechanism, this construct would still localize to the nucleus. It was found that both mRFP-PKM Apl III and mRFP-PKM Apl III D392A were in fact cytoplasmic after expression in sensory neurons; there was no nuclear enrichment in either case (Fig. 2B). Thus, kinase activity is required for nuclear export as well as cleavage and formation of PKM Apl III.

To summarize, overexpression of PKC Apl III in sensory neurons (or motor neurons) leads to cleavage and separation of the $\mathrm{N}$-terminal and C-terminal regions of the kinase (Bougie et al., 2009). Here, we found that reducing PKC Apl III kinase activity, either through addition of the C-terminal YFP tag, or using a D392A mutation, blocked this redistribution, suggesting that kinase activity is required for overexpression-induced cleavage. Since there was actually increased cleavage of the less active CFPPKC Apl III-YFP by purified calpain, this is not due to the inability of the inactive PKC Apl III to be cleaved, but instead it is likely that the requirement for activity is due to a kinase-dependent activation of the proteolytic process in cells.
Increasing kinase activity is sufficient to induce both nuclear export

and cleavage

If overexpression of PKC Apl III activates cleavage through kinase activation of a proteolytic event, then it should be possible to rescue this deficit of cleavage of the inactive $\mathrm{PKC}$ by coexpressing an active kinase. To test this, we coinjected CFP-PKC Apl III-YFP with the constitutively active mRFP-PKM Apl III in sensory neurons to determine whether this could lead to cleavage, measured either by loss of FRET or by increased cytoplasmic localization. As a control, we used the kinase-inactive mRFP-PKM Apl III D392A. Coexpression of the active mRFP-PKC Apl III with the FRET reporter CFP-PKC Apl III-YFP resulted in a significant loss of FRET compared with expressing the inactive MRFPPKM Apl III D392A with the FRET reporter [Fig. 3A; FRET values in neurons expressing active mRFP-PKM Apl III $(n=35$ neurons from three independent experiments) were $72 \pm 6 \%$ of the neurons expressing mRFP-PKM Apl III D392A ( $n=32$ neurons from three independent experiments); $p<0.01$, Student's $t$ test]. Expression of the active $\mathrm{mRFP}-\mathrm{PKC}$ Apl III also caused a significant redistribution of the YFP fluorescence (C-terminal marker) to the cytosol compared with neurons expressing the inactive $\mathrm{mR}$ FP-PKC Apl II D392A [Fig. 3B; nuclear/cytoplasmic ratios in neurons expressing active mRFP-PKM Apl III $(n=44$ cells from three independent experiments) was $80 \pm 3 \%$ of the ratio in neurons expressing either mRFP or mRFP PKC Apl III D392A ( $n=46$ cells from three independent experiments); $p<0.001$, Student's $t$ test; in this case, mRFP-PKM Apl II and mRFP results were combined as there were no significant differences between these two control groups]. Expression of the active mRFP-PKC Apl III also caused a smaller but significant redistribution of the CFP fluorescence (N-terminal marker) to the cytosol compared with neurons expressing the inactive mRFP-PKC Apl II D392A [Fig. 3B; nuclear/cytoplasmic ratios in neurons expressing active mRFP-PKM Apl III ( $n=44$ cells from three independent experiments) was $88 \pm 3 \%$ of the ratio in neurons expressing either mRFP or mRFP-PKC Apl III D392A; $p<0.01$, Student's $t$ test]. Both the loss of FRET and the increase in the cytoplasmic localization of the YFP fluorescence marking the $\mathrm{C}$ terminus with expression of the active mRFP-PKM Apl III indicate that increasing kinase activity within the cell is sufficient to induce cleavage of PKC Apl III. The increased cytosolic localization of the CFP fluorescence ( $\mathrm{N}$-terminal marker) is consistent with the role of kinase activity in nuclear export as well.

\section{5-HT does not induce cleavage of PKC Apl III in sensory neuron processes}

PKC is required for multiple forms of plasticity in the sensory neuron (Sossin, 2007). In particular, a 10 min application of 5-HT leads to a PKC-dependent form of ITF (Li et al., 2005; Villareal et al., 2007) that is blocked by inhibitors of PKC injected into the sensory neuron (Jin et al., 2011). To investigate whether 10 min of 5-HT was sufficient to induce cleavage of PKC Apl III into PKM Apl III, we injected low levels of the CFP-PKC Apl III-YFP into isolated Aplysia sensory neurons. Low levels were used to minimize any possible dominant-negative effect of the 


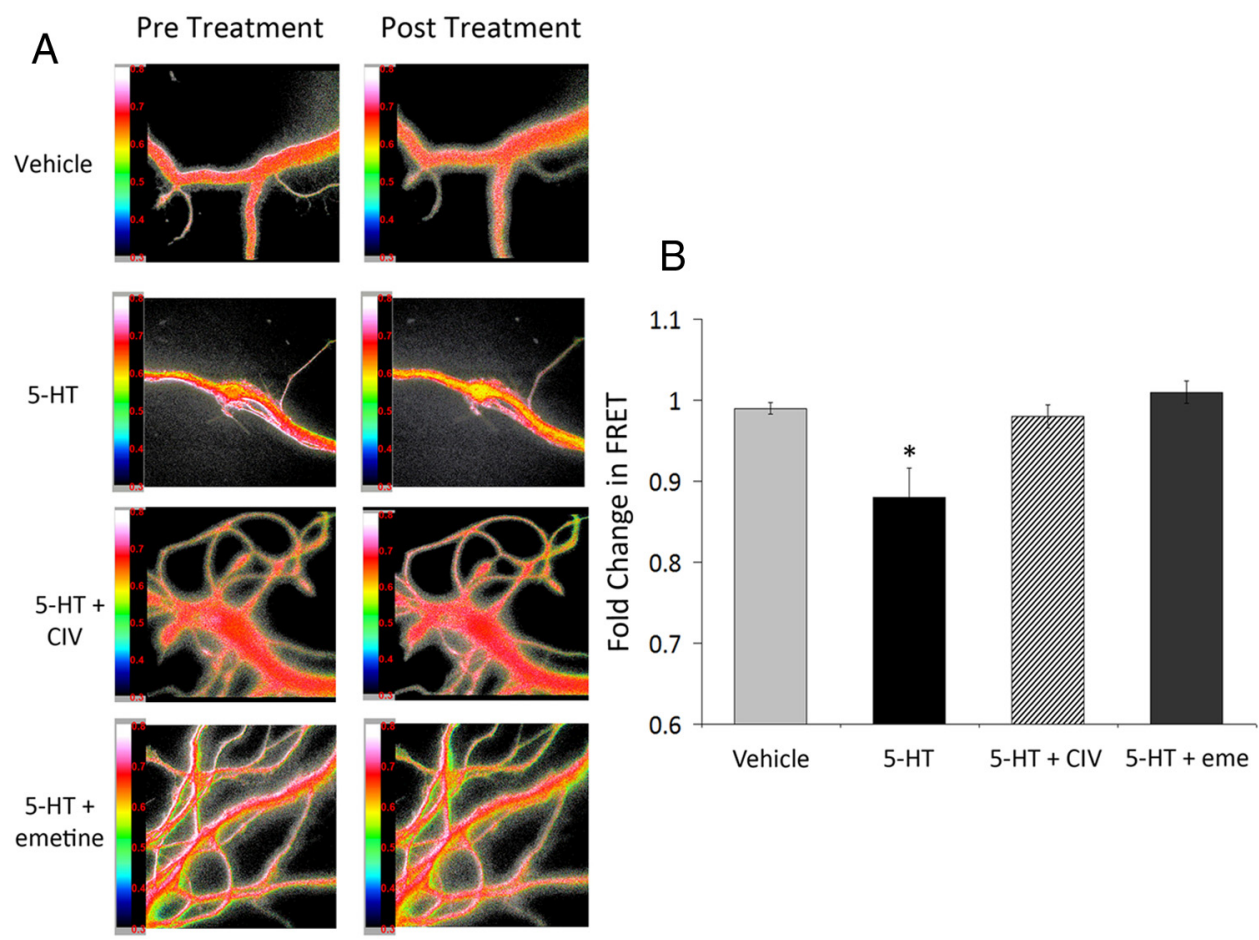

Figure 5. 5-HT induces cleavage of CFP-PKC ApI III-YFP in Aplysia motor neuron processes in a calpain- and protein synthesis-dependent manner. A, Color-coded FRET maps of Aplysia motor neuron processes expressing low levels of CFP-PKC ApI III-YFP, pretreatment and posttreatment. Scale is from 0.3 to 0.8 . The FRET maps display color-coded images of the measured FRET signal, in which warm colors represent higher levels of FRET and cooler colors represent lower levels of FRET (the gray color is unassigned and represents negative or undefined values sometimes seen in the periphery of expressing cells, or when no FRET is observed). Cleavage is indicated as a loss of FRET as seen in the 5 -HT-treated motor neuron process posttreatment. $\boldsymbol{B}$, Quantification of $\boldsymbol{A}$ : Fold change in FRET was calculated for cells treated with a vehicle solution $(0.99 \pm 0.01 ; n=10$ cells from four independent experiments), 5 -HT $(0.88 \pm 0.04 ; n=22$ cells from nine independent experiments), 5 -HT plus calpain inhibitor V ( $0.98 \pm 0.01 ; n=10$ cells from three independent experiments), or 5 -HT plus emetine (a protein synthesis inhibitor) $(1.01 \pm 0.01 ; n=9$ cells from three independent experiments). ${ }^{*} p<0.05$, Student's $t$ test with unequal variance using the Bonferroni correction for multiple tests. Error bars indicate SEM.

construct, since it has diminished activity compared with the endogenous PKC Apl III. At these low levels of expression, pigment granules in the cell body interfered with our FRET measurements; thus, we measured FRET in the sensory neuron processes, which lack pigment granules. Following injection, the sensory neurons were treated with $20 \mu \mathrm{M} 5-\mathrm{HT}$ for $10 \mathrm{~min}$, a protocol that can produce ITF, or a vehicle solution. The FRET values were then measured in the sensory neuron processes pretreatment and posttreatment. As can be seen by the FRET maps (Fig. $4 A$, quantified in $B$ ), there was no change in FRET signal after addition of 5 -HT in sensory neuron processes.

\section{5-HT induces calpain- and protein synthesis-dependent cleavage of PKC Apl III to PKM Apl III in Aplysia motor neuron processes}

We then repeated these experiments in motor neuron processes. Overexpression of mRFP-PKC Apl III also leads to cleavage in isolated motor neurons (Bougie et al., 2009). Moreover, a treatment with $20 \mu \mathrm{M} 5$-HT for $10 \mathrm{~min}$ in isolated motor neurons produces an enhancement of the postsynaptic glutamate response that is thought to be dependent on PKM Apl III production (Villareal et al., 2009). Similarly to the experiment in sensory neurons, we injected low levels of the CFP-PKC Apl III-YFP into isolated Aplysia LFS motor neurons. Following injection, the motor neurons were treated with $20 \mu \mathrm{M} 5$-HT for $10 \mathrm{~min}$ or a vehicle solution. The FRET values were then measured in the motor neuron processes pretreatment and posttreatment. As can be seen by the FRET maps, a small but significant decrease in the FRET ratio was detected in the cells treated with 5-HT, whereas no change was observed for the cells treated with the vehicle solution alone (Fig. $5 A$, quantified in $B$ ).

We then tested whether this 5-HT-induced cleavage of PKC Apl III was dependent on calpain, as we have previously shown for the cleavage seen after overexpression (Bougie et al., 2009). It is also known that calpain is required for the enhancement of the postsynaptic glutamate response (Villareal et al., 2009). Accordingly, cells injected with low levels of the CFP-PKC Apl III-YFP were pretreated with calpain inhibitor $\mathrm{V}$, a general and irreversible inhibitor of calpains, at $100 \mu \mathrm{M}$ for $10 \mathrm{~min}$ before application of 5-HT, or a vehicle solution. The pretreatment with the calpain inhibitor prevented the 5-HT-induced loss of FRET, indicating that the 5-HT-induced cleavage of PKC Apl III is dependent on calpain (Fig. 5A, quantified in $B$ ).

It has also been previously found that the 5-HT-induced enhancement of the glutamate response in Aplysia motor neurons is dependent on local protein synthesis and can be blocked with the protein synthesis inhibitor emetine (Villareal et al., 2007). Thus, we used this inhibitor to test whether the 5-HT-induced cleavage of PKC Apl III is also dependent on protein synthesis. The cells injected with CFP-PKC Apl III-YFP were either pretreated with emetine at $3 \mu \mathrm{M}$ for $10 \mathrm{~min}$ before 5-HT application, or pretreated with a vehicle solution alone. Inhibiting protein synthesis with emetine blocked the 5-HT-induced cleavage of CFP-PKC Apl III-YFP (Fig. 5A, quantified in $B$ ).

Thus, 5-HT-induced cleavage of the FRET construct in isolated motor neurons. Similar to the 5-HT-induced enhancement of the glutamate response, the loss of FRET was blocked by both calpain inhibitors and protein synthesis inhibitors. Our data sug- 
A
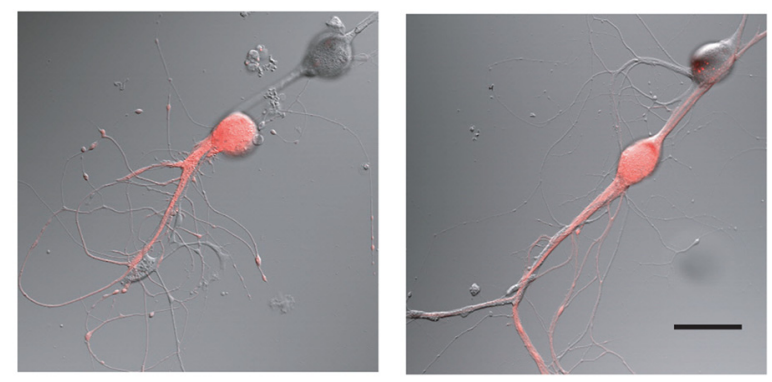

B

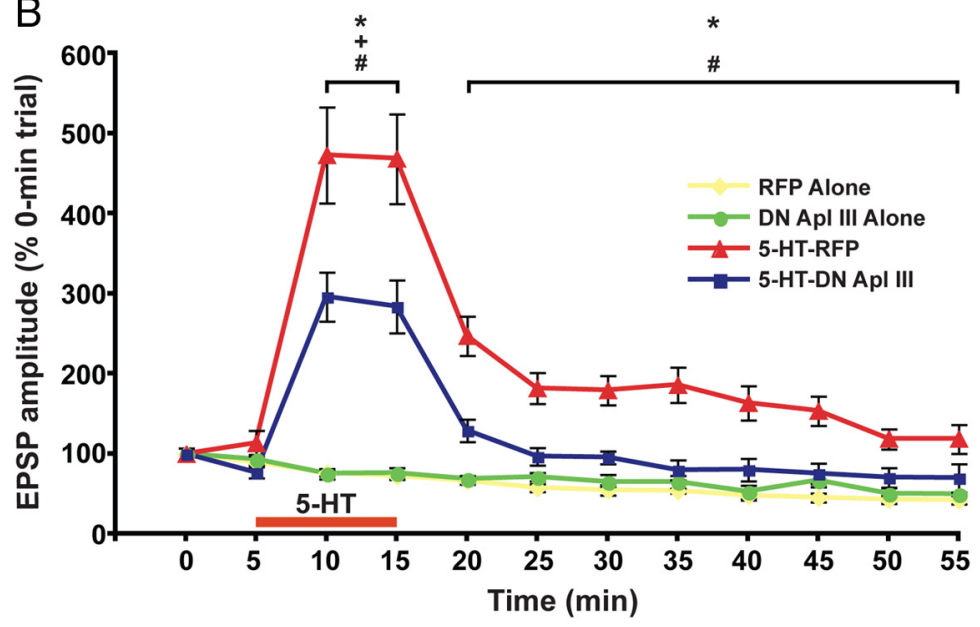

Figure 6. Overexpression of a dominant-negative version of PKC Apl III in the motor neuron disrupts maintenance of 5-HTdependent ITF of sensory-motor synapse in culture. $A$, Confocal fluorescence images of cocultures expressing mRFP alone (left) and mRFP-dominant-negative PKC Apl III (right). Two days after fabrication of sensory-motor cocultures, solutions of plasmid constructs in $\mathrm{dH}_{2} \mathrm{O}$ containing $0.25 \%$ fast green were pressure injected into the cell nucleus of the motor neuron. The cocultures were then imaged on a Zeiss Pascal scanning laser microscope after overnight incubation at $18^{\circ} \mathrm{C}$. Scale bar, $50 \mu \mathrm{m}$. B., Mean normalized amplitude of EPSPs in the four experimental groups: cocultures treated with 5-HT with postsynaptic overexpression of mRFP (5-HT-RFP, $n=7)$; cocultures treated with 5-HT with postsynaptic overexpression of dominant-negative PKC ApI III (5-HT-DN ApI $\mathrm{III}, n=5$ ); cocultures with mRFP overexpressed postsynaptically, but not treated with 5-HT (RFP Alone, $n=9$ ); cocultures with dominant-negative PKC Apl III overexpressed postsynaptically, but not treated with 5-HT (DN Apl III Alone, $n=6$ ). The 10 min period of 5-HT $(20 \mu \mathrm{m})$ treatment is indicated by the red bar. Repeated measures of ANOVA on the overall data from the RFP-Alone and DN ApI III Alone groups indicated that there was no significant difference between these two test-alone groups $\left(F_{(1,13)}=1.80\right.$; $p=0.20$ ). Accordingly, the data from RFP Alone and DN ApI III Alone experiments were combined for statistical comparisons with the two groups that received 5-HT treatment. A repeated-measures ANOVA on the data from the 5 - $\mathrm{HT}$ treatment period $(10-15$ min trials) indicated that there was a significant main effect for experimental treatment $\left(F_{(2,24)}=58.24 ; p<0.001\right)$. The mean normalized EPSPs for the $10 \mathrm{~min}$ trial were $472.8 \pm 60.0 \%$ in the 5 -HT-RFP group, $296.3 \pm 30.6 \%$ in the 5 -HT-DN Apl III group, $75.9 \pm 3.9 \%$ in the RFP-Alone group, and $74.9 \pm 6.3 \%$ in the DN-ApI III Alone group. The mean normalized EPSPs for the 15 min trial were $468.3 \pm 56.2 \%$ in the 5 -HT-RFP group, $283.6 \pm 33.1 \%$ in the 5 -HT-DN Apl III group, $72.0 \pm 4.2 \%$ in the RFP-Alone group, and $75.8 \pm 6.7 \%$ in the DN-Apl III Alone group. Tukey's HSD post hoc tests indicated that the EPSPs in the 5-HT-RFP group were significantly greater than those in the other two experimental groups during the $5-\mathrm{HT}$ treatment period $(p<0.05$ for each test), and that the EPSPs in the 5-HT-DN ApI III group were significantly greater than those in the combined test-alone group during 5 -HT treatment $(p<0.05)$. A repeated-measures ANOVA on the data from the period after washout of 5-HT ( $20-55$ min trials) revealed a highly significant main effect for the experimental treatment during this period $\left(F_{(2,24)}=46.81 ; p<0.001\right)$. Tukey's HSD post hoc tests showed that the mean value for the EPSPs after drug washout was significantly greater for the 5 -HT-RFP group than those for the other two experimental groups ( $p<0.05$ for each test). The difference between the EPSP data for the 5 -HT-DN Apl III and combined test-alone groups during this period, however, was not significant $(p=0.06)$. The asterisks (*) indicate significant differences between the 5-HT-RFP and test-alone data, the plus signs ( + ) indicate the significant difference between the 5-HT-DN Apl III and test-alone data, and the pound signs (\#) indicate significant differences between the 5-HT-RFP and 5-HT-DN ApI III data. Error bars represent \pm SEM. Interstimulus interval, $5 \mathrm{~min}$.

gest that the underlying mechanism for the enhancement of the glutamate response is cleavage of PKC Apl III into a PKM.

\section{A dominant-negative PKM Apl III blocks intermediate-term facilitation induced by 10 min of $5-\mathrm{HT}$}

The increase in the glutamate response is important for the late phase of ITF induced by 10 min of 5 -HT (Chitwood et al., 2001; Li et al., 2005). Indeed, this form of ITF, similar to the increase in the glutamate response, requires protein synthesis in the motor neuron (Villareal et al., 2007; Jin et al., 2011). To specifically test the role of PKM Apl III, we used the kinase-dead mRFP-PKM D392A as a dominant negative. Kinase-dead dominant negatives have been previously used to demonstrate specific roles for PKC Apl I and PKC Apl II (Manseau et al., 2001; Zhao et al., 2006; Lorenzetti et al., 2008; $\mathrm{Hu}$ et al., 2010). In motor neurons injected with the mRFP-PKM D392A, but not motor neurons injected with $\mathrm{mRFP}$, the later phase of ITF seen after a $10 \mathrm{~min}$ application of 5-HT was reduced (Fig. 6). These results demonstrate the physiological importance of PKM Apl III in the motor neuron for the late phase of ITF seen after 10 min of 5-HT.

\section{Discussion}

\section{Conservation of the role of an atypical} PKM in the memory trace

In vertebrates, inhibitors of atypical PKMs permanently erase long-term memory at time points when a given trace is expected to be firmly consolidated (Pastalkova et al., 2006; Shema et al., 2007, 2011). The evidence that inhibiting atypical PKMs erases memory is based on two inhibitors of PKM, ZIP and chelerythrine (for review, see Sacktor, 2011). Memory is also blocked by the expression of a dominant-negative PKM $\zeta$ (Ling et al., 2002; Shema et al., 2011), and overexpression of $\mathrm{PKM} \zeta$ can enhance longterm memory in both Drosophila and rodents (Drier et al., 2002; Shema et al., 2011). It has also been recently shown that the role of PKM is conserved in Aplysia, as inhibiting the atypical PKM in Aplysia, PKM Apl III, using either ZIP or chelerythrine erases the long-term memory of behavioral sensitization, in addition to erasing the long-term enhancement of synaptic plasticity, which is the cellular correlate of this behavioral memory (Cai et al., 2011).

A missing step in Aplysia has been evidence for the formation of PKM during learning. We have proposed that cleavage by calpain is the mechanism of PKM formation. Previous evidence in favor of this includes the blockade of some forms of plasticity by calpain inhibitors (Sutton et al., 2004; Villareal et al., 2009) and evidence that mRFP-PKC Apl III can be cleaved by calpain when overexpressed

(Bougie et al., 2009).

In the present study, we have provided evidence that PKC Apl III is cleaved by 5 -HT during a form of synaptic plasticity related to memory (ITF) and shown that the requirements for cleavage are the same as the requirements for the plasticity. Specifically, we have developed a FRET reporter to measure cleavage of PKC Apl III in living neurons and have applied it to demonstrate 5-HT- 

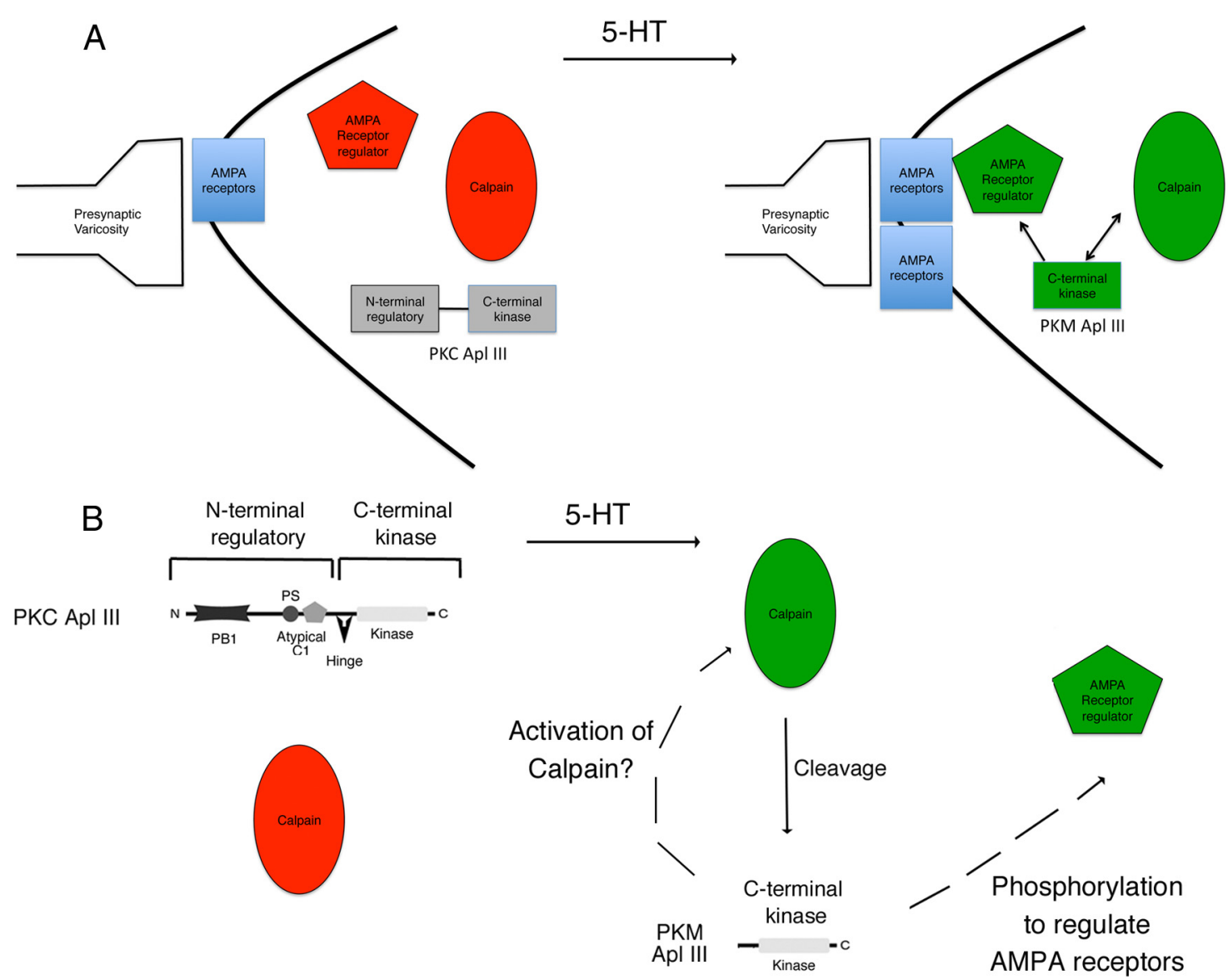

Figure 7. Schematic of PKC ApI III cleavage and regulation of AMPA receptors. A spatial schematic $(\boldsymbol{A})$ and a more detailed nonspatial schematic $(\boldsymbol{B})$ of the proposed mechanism for plasticity are shown. In the postsynaptic process, calpain, PKC ApI III, AMPA receptors, and an AMPA receptor regulator are all present. After 5-HT, calpains are activated in a protein synthesis-dependent manner, leading to the cleavage of PKC ApI III into PKM ApI III. We propose that PKM Apl III then phosphorylates an AMPA receptor regulator to increase the number of AMPA receptors at the synapse. We also propose that PKM ApI III can activate calpain in a 5-HT-independent process, leading to additional cleavage of PKC ApI III. Activation of calpain, PKM ApI III, and phosphorylation of the AMPA receptor regulator are shown by the green color.

mediated, calpain- and protein synthesis-dependent, cleavage of PKC Apl III in isolated Aplysia motor neurons. We propose a model whereby 5-HT induces association of the calpain and PKC Apl III, followed by activation of the calpain and cleavage of PKC Apl III into PKM Apl III, where it then acts on regulators of AMPA receptors (Fig. 7).

In vertebrates, $\mathrm{PKM} \zeta$ formation is thought to be due to increased translation of a specific transcript encoding $\operatorname{PKM} \zeta$, as opposed to calpain-mediated cleavage. Indeed, there is evidence for increased translation of the alternative transcript during memory formation (Hernandez et al., 2003). However, it should be noted that it has not yet been shown that specific blockade of translation of the PKM $\zeta$ message blocks either the induction or the maintenance of memory, and thus a role for calpainmediated cleavage in vertebrates cannot be ruled out at this point.

\section{Why is kinase activity required to induce formation of a PKM?}

We have shown that kinase activity is necessary (Fig. 2) and sufficient for cleavage of overexpressed PKC Apl III (Fig. 3). It is possible that kinase activity is required to activate calpain in order for the cleavage to occur. Indeed, mammalian calpain can be activated in neurons through phosphorylation by ERK (Zadran et al., 2010) and ERK is activated by 5-HT in Aplysia and is important for some forms of plasticity and memory (Sharma and Carew, 2004). It is also possible that the kinase must phosphory- late an additional target that subsequently activates or recruits calpain. However, the amount of cleavage observed after expressing the active PKM with the inactive CFP-PKC Apl III-YFP was less than seen after overexpression, where it appeared that most of the overexpressed kinase is cleaved. It may be that normally activation occurs in a complex in which the kinase to be cleaved directly activates the calpain that cleaves it, and thus the transeffect of adding PKM is not as strong. It is also possible that protein-protein interactions involving the full-length $\mathrm{PKC}$ are important for targeting the calpain or associated protein for phosphorylation, again explaining the decreased ability of PKM to stimulate cleavage in trans.

The fact that activity appears to be involved in recruitment of the calpain suggests a hypothetical positive-feedback pathway that may be important for the maintenance of PKM activation (Fig. 7). Following 5-HT-induced calpain-mediated cleavage of PKC Apl III into PKM Apl III, we hypothesize that PKM Apl III could recruit calpain in a 5-HT-independent manner to induce additional cleavage of PKC Apl III to maintain levels of PKM.

\section{Is the kinase dependence for cleavage downstream of a kinase} dependence for nuclear export?

The inactive mRFP-PKC Apl III D392A was enriched in the nucleus, suggesting that kinase activity is also required for nuclear export of the protein. Ideally, we could mutate the nuclear localization sequence in this context and then determine whether the 
kinase-dead cytoplasmic protein was still cleaved. However, while putative nuclear import and export sites were identified for vertebrate PKCı (Perander et al., 2001), we did not observe differences in nuclear localization after mutating these sites (data not shown). Expression of mRFP-PKC Apl III in SF9 cells did not lead to nuclear localization (Bougie et al., 2009), suggesting specific factors in Aplysia neurons may be required for nuclear import; however, expression of kinase-dead mRFP-PKC Apl III D392A in SF9 cells did in fact lead to nuclear retention (data not shown), suggesting that this may be more of a quantitative than qualitative difference. It is possible that PKC Apl III may "piggyback" into the nucleus through binding to another protein that has a nuclear localization sequence. This binding would involve an $\mathrm{N}$-terminal sequence. Perhaps this binding is removed by PKC Apl III phosphorylation of this protein, allowing the kinase to shuttle back to the cytoplasm. The identity of these potential binding proteins is currently unknown, but may present an interesting opportunity for further investigation.

\section{PKM Apl III is formed following facilitating stimuli}

We have shown that 5-HT is able to induce cleavage of PKC Apl III into PKM Apl III in isolated Aplysia motor neuron processes (Fig. 5). It has been previously established that a 10 min treatment of isolated motor neurons with 5-HT produces an enhancement of the postsynaptic glutamate response that is thought to be dependent on PKM Apl III production (Villareal et al., 2009). Here, we show that the dominant-negative PKM Apl III blocked ITF after $10 \mathrm{~min}$ of 5-HT treatment, consistent with a role for the enhanced response to glutamate in ITF. However, it cannot be ruled out that the role of the PKM Apl III in the motor neuron is to generate a retrograde signal required for activation of the sensory neuron. We did not detect cleavage of the CFP-PKC Apl III-YFP construct in isolated sensory neurons. However, we cannot eliminate the possibility that this occurs during ITF in which motor neurons may provide a retrograde signal required for cleavage. Indeed, some important plasticity-related events in the sensory neuron, such as the translational regulation of sensorin during activity-dependent long-term facilitation (LTF), require the motor neuron to be present to induce plasticity occurring in the sensory neuron (Cai et al., 2008; Wang et al., 2009).

The amount of cleavage we detected in the motor neuron was small ( $\mathrm{a} \sim 10 \%$ decrease in FRET). This may be expected, due to the fact that $10 \mathrm{~min}$ of 5-HT does not lead to a long-lasting plasticity at sensory-motor neuron synapses. It has recently been shown that both the cellular LTF as well as behavioral long-term memory in Aplysia is maintained by PKM Apl III-dependent protein phosphorylation (Cai et al., 2011). Thus, it is likely that calpain-dependent cleavage is also involved in long-term memory in Aplysia. It will be interesting to determine in the future if the amount of cleavage is greater during LTF formation. Since LTF plasticity is dependent on transcription, it may be that additional cleavage in this case will be delayed and dependent on transcriptional products.

\section{Cleavage of the CFP-PKC Apl III-YFP requires protein synthesis}

While it is known that 5-HT induces dendritic protein translation (Villareal et al., 2007), it is not known which proteins are synthesized to induce cleavage of PKC Apl III. It is possible that it is in fact calpain that needs to be synthesized de novo to cleave PKC Apl III into PKM Apl III, or alternatively, that the role of the new protein synthesized could be to recruit calpain to cleave PKC Apl III. Since PKC Apl III is cleaved when expressed at high levels, an attractive hypothesis is that the protein translated is PKC Apl III itself and the increase in the levels of the protein activate the cleavage. However, our experiments argue against this, since cleavage is still protein synthesis dependent even when CFP-PKC Apl III-YFP is overexpressed.

\section{Conclusion}

In the present study, we have provided evidence that PKC Apl III is cleaved into PKM Apl III during memory formation, and the requirements for cleavage are the same as the requirements for the plasticity. Thus, our data further support the hypothesis that the PKM required for formation of the memory trace is formed by calpain-dependent cleavage in Aplysia.

\section{References}

Bougie JK, Lim T, Farah CA, Manjunath V, Nagakura I, Ferraro GB, Sossin WS (2009) The atypical protein kinase C in Aplysia can form a protein kinase M by cleavage. J Neurochem 109:1129-1143.

Cai D, Chen S, Glanzman DL (2008) Postsynaptic regulation of long-term facilitation in Aplysia. Curr Biol 18:920-925.

Cai D, Pearce K, Chen S, Glanzman DL (2011) Protein kinase M maintains long-term sensitization and long-term facilitation in Aplysia. J Neurosci 31:6421-6431.

Cameron AJ, Escribano C, Saurin AT, Kostelecky B, Parker PJ (2009) PKC maturation is promoted by nucleotide pocket occupation independently of intrinsic kinase activity. Nat Struct Mol Biol 16:624-630.

Chitwood RA, Li Q, Glanzman DL (2001) Serotonin facilitates AMPA-type responses in isolated siphon motor neurons of Aplysia in culture. J Physiol 534:501-510.

Chou MM, Hou W, Johnson J, Graham LK, Lee MH, Chen CS, Newton AC, Schaffhausen BS, Toker A (1998) Regulation of protein kinase C $\zeta$ by PI 3-kinase and PDK-1. Curr Biol 8:1069-1077.

Drier EA, Tello MK, Cowan M, Wu P, Blace N, Sacktor TC, Yin JC (2002) Memory enhancement and formation by atypical PKM activity in Drosophila melanogaster. Nat Neurosci 5:316-324.

Hernandez AI, Blace N, Crary JF, Serrano PA, Leitges M, Libien JM, Weinstein G, Tcherapanov A, Sacktor TC (2003) Protein kinase M $\zeta$ synthesis from a brain mRNA encoding an independent protein kinase $\mathrm{C} \zeta$ catalytic domain: implications for the molecular mechanism of memory. J Biol Chem 278:40305-40316.

Hu JY, Chen Y, Bougie JK, Sossin WS, Schacher S (2010) Aplysia cell adhesion molecule and a novel protein kinase $\mathrm{C}$ activity in the postsynaptic neuron are required for presynaptic growth and initial formation of specific synapses. J Neurosci 30:8353-8366.

Jin I, Kandel ER, Hawkins RD (2011) Whereas short-term facilitation is presynaptic, intermediate-term facilitation involves both presynaptic and postsynaptic protein kinases and protein synthesis. Learn Mem 18:96102.

Kandel ER (2001) Nobel Lecture: the molecular biology of memory storage: a dialog between genes and synapses. Biosci Rep 21:565-611.

Li Q, Roberts AC, Glanzman DL (2005) Synaptic facilitation and behavioral dishabituation in Aplysia: dependence on release of $\mathrm{Ca}^{2+}$ from postsynaptic intracellular stores, postsynaptic exocytosis, and modulation of postsynaptic AMPA receptor efficacy. J Neurosci 25:5623-5637.

Lim T, Sossin WS (2006) Phosphorylation at the hydrophobic site of protein kinase C Apl II is increased during intermediate term facilitation. Neuroscience 141:277-285.

Ling DS, Benardo LS, Serrano PA, Blace N, Kelly MT, Crary JF, Sacktor TC (2002) Protein kinase $\mathrm{M} \zeta$ is necessary and sufficient for LTP maintenance. Nat Neurosci 5:295-296.

Lorenzetti FD, Baxter DA, Byrne JH (2008) Molecular mechanisms underlying a cellular analog of operant reward learning. Neuron 59:815-828.

Manseau F, Fan X, Hueftlein T, Sossin W, Castellucci VF (2001) $\mathrm{Ca}^{2+}$ independent protein kinase C Apl II mediates the serotonin-induced facilitation at depressed Aplysia sensorimotor synapses. J Neurosci 21: 1247-1256.

Marinesco S, Carew TJ (2002) Serotonin release evoked by tail nerve stimulation in the CNS of Aplysia: characterization and relationship to heterosynaptic plasticity. J Neurosci 22:2299-2312. 
Montarolo PG, Goelet P, Castellucci VF, Morgan J, Kandel ER, Schacher S (1986) A critical period for macromolecular synthesis in long-term heterosynaptic facilitation in Aplysia. Science 234:1249-1254.

Pastalkova E, Serrano P, Pinkhasova D, Wallace E, Fenton AA, Sacktor TC (2006) Storage of spatial information by the maintenance mechanism of LTP. Science 313:1141-1144.

Perander M, Bjorkoy G, Johansen T (2001) Nuclear import and export signals enable rapid nucleocytoplasmic shuttling of the atypical protein kinase $\mathrm{C} \lambda$. J Biol Chem 276:13015-13024.

Roberts AC, Glanzman DL (2003) Learning in Aplysia: looking at synaptic plasticity from both sides. Trends Neurosci 26:662-670.

Sacktor TC (2011) How does PKM $\zeta$ maintain long-term memory? Nat Rev Neurosci 12:9-15.

Serrano P, Yao Y, Sacktor TC (2005) Persistent phosphorylation by protein kinase $\mathrm{M} \zeta$ maintains late-phase long-term potentiation. J Neurosci 25:1979-1984.

Sharma SK, Carew TJ (2004) The roles of MAPK cascades in synaptic plasticity and memory in Aplysia: facilitatory effects and inhibitory constraints. Learn Mem 11:373-378.

Shema R, Sacktor TC, Dudai Y (2007) Rapid erasure of long-term memory associations in the cortex by an inhibitor of PKM $\zeta$. Science 317:951-953.

Shema R, Haramati S, Ron S, Hazvi S, Chen A, Sacktor TC, Dudai Y (2011) Enhancement of consolidated long-term memory by overexpression of protein kinase $\mathrm{M} \zeta$ in the neocortex. Science 331:1207-1210.

Si K, Giustetto M, Etkin A, Hsu R, Janisiewicz AM, Miniaci MC, Kim JH, Zhu $\mathrm{H}$, Kandel ER (2003) A neuronal isoform of CPEB regulates local protein synthesis and stabilizes synapse-specific long-term facilitation in Aplysia. Cell 115:893-904.
Sossin WS (2007) Isoform specificity of protein kinase Cs in synaptic plasticity. Learn Mem 14:236-46.

Sossin WS (2008) Defining memories by their distinct molecular traces. Trends Neurosci 31:170-175.

Sutton MA, Bagnalll MW, Sharma SK, Shobe J, Carew TJ (2004) Intermediate-term memory for site-specific sensitization in Aplysia is maintained by persistent activation of protein kinase C. J Neurosci 24:3600-3609.

Villareal G, Li Q, Cai D, Glanzman DL (2007) The role of rapid, local, postsynaptic protein synthesis in learning-related synaptic facilitation in Aplysia. Curr Biol 17:2073-2080.

Villareal G, Li Q, Cai D, Fink AE, Lim T, Bougie JK, Sossin WS, Glanzman DL (2009) Role of protein kinase $\mathrm{C}$ in the induction and maintenance of serotonin-dependent enhancement of the glutamate response in isolated siphon motor neurons of Aplysia californica. J Neurosci 29:5100-5107.

Wang DO, Kim SM, Zhao Y, Hwang H, Miura SK, Sossin WS, Martin KC (2009) Synapse- and stimulus-specific local translation during longterm neuronal plasticity. Science 324:1536-1540.

Xia Z, Liu Y (2001) Reliable and global measurement of fluorescence resonance energy transfer using fluorescence microscopes. Biophys J 81:2395-2402.

Zadran S, Jourdi H, Rostamiani K, Qin Q, Bi X, Baudry M (2010) Brain-derived neurotrophic factor and epidermal growth factor activate neuronal $\mathrm{m}$-calpain via mitogen-activated protein kinase-dependent phosphorylation. J Neurosci 30:1086-1095.

Zhao Y, Leal K, Abi-Farah C, Martin KC, Sossin WS, Klein M (2006) Isoform specificity of PKC translocation in living Aplysia sensory neurons and a role for $\mathrm{Ca}^{2+}$-dependent PKC APL I in the induction of intermediate-term facilitation. J Neurosci 26:8847-8856. 